\title{
Effect of increased body mass index (BMI) on time to tumour progression (TTP) in unresectable metastatic colorectal cancer (mCRC) patients treated with bevacizumab-based therapy
}

\author{
N. Faruk Aykan · Ibrahim Yildiz · Fatma Sen • \\ Leyla Kilic · Serkan Keskin · Rumeysa Ciftci • \\ Senem Karabulut $\cdot$ Burak Sakar $\cdot$ Rian Disci
}

Received: 27 June 2013/Accepted: 24 July 2013/Published online: 7 August 2013

(C) The Author(s) 2013. This article is published with open access at Springerlink.com

\begin{abstract}
High BMI is a well-known risk factor for the development and recurrence of several solid tumours, including CRC. Obesity is associated with increased levels of vascular endothelial growth factor (VEGF). Bevacizumab is the main targeted therapy for inhibiting tumour angiogenesis by blocking the VEGF/VEGF receptor pathway. Elevated VEGF in obese patients might provoke resistance to anti-VEGF therapy. We evaluated the efficacy of bevacizumab on TTP among mCRC patients through stratifying them according to their BMI. Patients with mCRC who had been treated with fluoropyrimidine-based combination chemotherapy with bevacizumab were included in the study. Patients were assigned according to their BMI before initiation of therapy (group A: BMI $<25 \mathrm{~kg} / \mathrm{m}^{2}$, group B: BMI $\geq 25 \mathrm{~kg} / \mathrm{m}^{2}$ ). Multivariate analysis was performed to evaluate the risk of tumour progression. Between April 2007 and June 2011, 80 patients were treated with chemotherapy and bevacizumab as first-line therapy $(n=37$ for group A, $n=43$ for group B). Tumours in $56.3 \%$ of the patients in group $\mathrm{A}(n=21)$ and $76.3 \%$ of the patients in group $\mathrm{B}(n=33)$ progressed during a median 10-months (3-57 months) follow-up. The median TTP was 11.7 months in the group A and 6 months in the group B $(p=0.004)$. In a multivariate analysis, high BMI $\left(\geq 25 \mathrm{~kg} / \mathrm{m}^{2}\right)$ was associated with significantly shorter TTP ( $p=0.01$; HR: 4.37). High
\end{abstract}

N. Faruk Aykan ( $)$ - I. Yildiz · F. Sen · L. Kilic · S. Keskin ·

R. Ciftci · S. Karabulut · B. Sakar

Department of Medical Oncology, Institute of Oncology,

Istanbul University, Istanbul, Turkey

e-mail:nfaruk@mac.com

R. Disci

Department of Biostatistics, Institute of Oncology, Istanbul

University, Istanbul, Turkey
BMI among mCRC patients treated with bevacizumab is associated with shorter TTP. Further study in larger databases is warranted for confirming the negative prognostic effect of obesity during treatment with anti-VEGF agents.

Keywords Colorectal cancer - Body mass index · Bevacizumab - Time to progression

\section{Introduction}

In recent decades, the prevalence of obesity has increased worldwide in all age groups [1]. Increasing epidemiological evidence has demonstrated that obesity is associated with an increased risk of cancer, especially colon cancer [2-4]. Several factors, such as insulin resistance, increased levels of leptin, plasminogen activator inhibitor-1, endogenous sex steroids, decreased levels of adiponectin, and chronic inflammation, are involved in carcinogenesis and cancer progression [5].

However, a few studies that have investigated the influence of BMI on the outcomes of colon cancer patients have reported inconsistent findings [6-11]. Despite support for the importance of obesity and metabolic syndrome as risk factors for the development of colorectal cancer, data are equivocal for the effects on colorectal cancer progression and outcome [6-8]. Several studies have reported decreased survival and increased recurrence in patients with insulin resistance or high BMI [7-9], whereas other studies have not [6, 10, 11]. Although high BMI was associated with better median OS in the CAIRO study, CAIRO2 study did not show this association [11]. Consequently, testing the role of BMI in patients receiving targeted therapy is an actual subject of research. 
Bevacizumab is a humanised monoclonal antibody (mAb) against vascular endothelial growth factor (VEGF), which is the major mediator of angiogenesis. Activation of the VEGF/VEGF receptor axis triggers multiple signalling networks responsible for endothelial cell survival, mitogenesis, migration, and differentiation [12]. Elevated serum VEGF levels have been observed in overweight or obese patients [13, 14]. The BMI of patients with metastatic CRC at treatment initiation predicts treatment outcomes. Guiu et al. [15] reported that high visceral fat area (VFA) measured by computed tomography independently predicted poorer outcome in a retrospective series of patients given first-line bevacizumab-based therapy for metastatic colon cancer.

Although not all patients diagnosed with metastatic CRC benefit from anti-VEGF antibody treatment, there are currently no biomarkers available to predict the efficacy of anti-angiogenic therapies. VEGF plays an important role as an endothelial mitogen in tumour angiogenesis, and increased levels of VEGF may contribute to poorer outcomes in cancer in obese subjects [16]. Thus, BMI may be a prognostic factor for poor outcomes in metastatic CRC patients receiving bevacizumab-based treatment. On the other hand, standard bevacizumab dose may be questionable for obese patients. We performed a study of the associations between BMI and clinical outcomes in consecutive patients administered bevacizumab-based treatments for metastatic CRC.

\section{Patients and methods}

\section{Eligible patients}

From April 2007 to June 2011, 80 consecutive patients with metastatic colorectal adenocarcinoma treated with fluoropyrimidine-based combination chemotherapy plus bevacizumab at the Institute of Oncology, Istanbul University (Istanbul, Turkey), were included in the study. Patients, age between 18 and 80 years old with histologically confirmed locally advanced or metastatic CRC, were determined. Those with one or more unidimensionally measurable lesions, who were not amenable to curative resection, with an Eastern Cooperative Oncology Group (ECOG) performance status of $0-2$, no prior history of cancer (except basal cell carcinoma or carcinoma in situ of the cervix), adequate bone marrow (white absolute neutrophil count, $\geq 1,500 / \mathrm{mm}^{3}$, platelet count $\geq 100,000 / \mathrm{mm}^{3}$ ) and liver function (total bilirubin $\leq 1.5 \mathrm{mg} / \mathrm{dl}$ and alanine and aspartate transaminase levels $<5 \mathrm{X}$ upper limit of normal), and a life expectancy of longer than 3 months were included. Patients who received bevacizumab as a second-line treatment were also included. Patients received one of the following treatment regimens: simplified LV5FU2 (leucovorin $400 \mathrm{mg} / \mathrm{m}^{2}$, followed by 5-fluorouracil as a $400 \mathrm{mg} / \mathrm{m}^{2}$ bolus and a $2,400 \mathrm{mg} / \mathrm{m}^{2}$ infusion over $46 \mathrm{~h}$ ), modified FOLFOX regimen (simplified LV5FU2 regimen plus oxaliplatin $85 \mathrm{mg} / \mathrm{m}^{2}$ ), FOLFIRI (simplified LV5FU2 regimen plus irinotecan $180 \mathrm{mg} / \mathrm{m}^{2}$ every 2 weeks), XELOX (capecitabine $1,000 \mathrm{mg} / \mathrm{m}^{2}$, b.i.d., p.o., days $1-14$ plus oxaliplatin $130 \mathrm{mg} / \mathrm{m}^{2}$ every 3 weeks), or XELIRI (capecitabine $1,000 \mathrm{mg} / \mathrm{m}^{2}$, b.i.d., p.o., days $1-14$ plus irinotecan $240 \mathrm{mg} / \mathrm{m}^{2}$ every 3 weeks). Bevacizumab was administered at a dosage of $5 \mathrm{mg} / \mathrm{kg}$ every 2 weeks or $7.5 \mathrm{mg} / \mathrm{kg}$ every 3 weeks. The dosages of chemotherapeutic agents were determined according to the measured body surface area (BSA); however, for patients with BSA over $2 \mathrm{~m}^{2}$, dosages were adjusted according to $2 \mathrm{~m}^{2}$. For bevacizumab, no dosing reduction was accomplished, i.e. patients received the exact planned dose for their actual weight. All of the patients had unresectable liver metastases associated with primary tumours at the initial consultation. Patients who underwent hepatic resection and local ablative therapies, chemo-embolisation, or radio-embolisation were excluded. Pregnant or breastfeeding women were excluded. Other key exclusion criteria were as follows: clinically significant cardiovascular disease; exclusive bone metastasis; use of full-dose anticoagulants or thrombolytics; known CNS metastases; serious non-healing wound, ulcer, clinically significant bleeding diathesis, or coagulopathy.

Tumour response was evaluated using a computed tomography scan or magnetic resonance imaging depending on which imaging methods were used at baseline. Tumour responses and progression were determined every 8 weeks using the Response Evaluation Criteria in Solid Tumours [17] by the investigators and classified as follows: complete response (CR), partial response (PR), stable disease (SD), or progressive disease (PD). The tumour response after 2 months of chemotherapy was used for statistical analysis. Patients with either CR or PR were classified as responders, and patients with SD or PD were considered non-responders. This retrospective study was approved by our institutional review board.

Baseline demographic, clinical, and laboratory data, including age, gender, performance status, tumour marker levels, K-ras mutation status, and treatment details, were collected retrospectively for all patients using uniform database templates to ensure consistent data collection.

Statistical analyses

SPSS version 16.0 (SPSS Inc., Chicago, IL) was used for the statistical analyses. The follow-up duration was calculated from the date of the first bevacizumab administration to the date of death or last follow-up visit. The time 
to progression (TTP) was calculated as the period from the initiation of treatment to the first observation of disease progression or to disease-specific death. The influence of putative prognostic factors on the progression of disease was evaluated by univariate analysis. Fifty-four $(67.5 \%)$ of the 80 patients with complete clinical and pathological data experienced disease progression during follow-up. TTP was evaluated with Mann-Whitney U test. Descriptive, univariate, and multivariate analyses were performed for those 54 patients with progression. Univariate Cox proportional-hazards models of all the potential baseline predictors were built to compute the hazard ratios (HR) with $95 \%$ confidence intervals (CI). A multivariable binary logistic regression analysis was accomplished using a stepwise algorithm including age (years; $<60$ vs. $\geq 60$ ), BMI ( $<25$ vs. $\geq 25$ ), primary tumour status (intact versus non-intact), type of chemotherapy regimens (irinotecan based versus oxaliplatin based), K-ras mutational status (wild type versus mutated), carcinoembryonic antigen (CEA) level (median, $<28$ vs. $\geq 28$ ), and number of disease sites. For BMI, patients were categorised as underweight/ normal weight $\left(\mathrm{BMI}<25 \mathrm{~kg} / \mathrm{m}^{2}\right.$ ), or overweight/obese $\left(\mathrm{BMI} \geq 25 \mathrm{~kg} / \mathrm{m}^{2}\right)$. All $\mathrm{P}$ values were two-sided.

\section{Results}

At the time of the final follow-up, 4 (5\%) within 54 patients $(67.5 \%)$ who exhibited tumour progression were deceased due to disease-related factors. Remaining 26 $(32.5 \%)$ patients had no evidence of progression during the last evaluation.

The clinical and demographic data of 54 metastatic CRC patients who had disease progression during chemotherapy with bevacizumab were summarised in Table 1 and 2 . The median age for these patients was 60.5 years (range $34-78) ; 59.3 \%(n=32)$ of the patients were male, and $40.7 \%(n=22)$ were female. Distant metastasis was present in $63 \%$ of the patients at the time of diagnosis. Adjuvant chemotherapy was administered in $20(37.0 \%)$ patients. The primary location of the malignancy was sigmoid colon and recto-sigmoid junction in 18 patients $(33.4 \%)$, rectum in 17 patients $(31.5 \%)$, right colon-caecum in 14 patients $(25.8 \%)$, left colon in 3 patients $(5.6 \%)$, and transverse colon in two patients $(3.7 \%)$. Twenty-nine $(53.7 \%)$ patients had K-ras mutations, and 25 (46.3\%) patients had no mutations.

All patients received bevacizumab-containing chemotherapy regimens as first-line therapy. The median number of treatment cycles was 10 (range 2-32). Oxaliplatin-based chemotherapy was administered to 19 patients (35.2\%), and irinotecan-based chemotherapy was administered to 35 patients $(64.8 \%)$ in combination with bevacizumab.
Height and weight were recorded before initiation of bevacizumab and used to assign patients to group $\mathrm{A}$ $\left(\mathrm{BMI}<25 \mathrm{~kg} / \mathrm{m}^{2}\right)$ and group $\mathrm{B} \quad\left(\mathrm{BMI} \geq 25 \mathrm{~kg} / \mathrm{m}^{2}\right)$. Baseline characteristics (age, gender, number of metastatic locations, CEA, and CA 19-9 levels and K-ras mutation status) and treatment details such as number of cycles, resection status of the primary tumour, type of chemotherapeutic agent combined with bevacizumab, and response to treatment of all patients are represented in Tables 1 and 2. Twenty-one $(56.3 \%)$ of 37 patients in group A and $33(76.7 \%)$ of 43 patients in group B progressed during a median of 10-months follow-up (range 3-57 months).

For 54 patients who had disease progression and complete clinical data concerning the variables included in univariate analysis, the influence of the 7 variables on TTP was evaluated with Mann-Whitney U test. Finally through grouping TTP according to the median value, the effect of these variables on TTP was analysed with binary logistic regression model. In univariate analysis, $\mathrm{BMI} \geq 25$ $(p=0.004)$ and higher number of metastatic sites $(p=0.032)$ were associated with poor TTP. The median TTP was 11.7 months in the group A and 6 months in the group B $(p=0.004)$.

A multivariate logistic regression model for TTP was constructed that included age, BMI, serum CEA level, K-ras mutational status, primary tumour status (intact or non-intact), chemotherapy regimens, and number of disease sites. The results are displayed in Table 3 . The only independent prognostic factor for TTP was BMI ( $p=0.01$; OR, 4.37; $95 \%$ CI, 1.34-14.78) for patients with metastatic CRC treated with bevacizumab.

\section{Discussion}

Recent phase III trials have shown that adding bevacizumab to a first-line conventional chemotherapeutic regimen improved progression-free survival and overall survival in patients with metastatic CRC [18, 19]. Despite extensive investigation, there are no validated predictive biomarkers of the efficacy of VEGF-targeted therapies, such as bevacizumab. Obese animal models have been shown to be resistant to anti-VEGF treatment [20], which suggests that increased amounts of visceral fat may be associated with high VEGF levels and resistance to bevacizumab-based regimens in patients with metastatic CRC [15].

In the present study, the median TTP was 11.7 months in the BMI $<25$ group and 6 months in the BMI $\geq 25$ group ( $p=0.004)$. In addition, the multivariate analysis indicated that increased BMI was the most important predictor of progression in the patients receiving the bevacizumab treatment. Evidence accumulated over the past decade has 
Table 1 Basal characteristics of metastatic CRC patients who progressed during bevacizumab-containing chemotherapy

\begin{tabular}{|c|c|c|c|}
\hline Characteristic & $\begin{array}{l}\text { Patient } \\
\text { number }(\%)\end{array}$ & Characteristic & \\
\hline$B M I$ & & Age & \\
\hline $\mathrm{BMI}<25$ & $21(38.9)$ & Median (years) & 60.5 \\
\hline $\mathrm{BMI} \geq 25$ & $33(61.1)$ & (range) & $(34-78)$ \\
\hline Sex & & $C E A$ & \\
\hline Male & $32(59.3)$ & Median (ng/ml) & 5 \\
\hline Female & $22(40.7)$ & (range) & $(0.8-20,300)$ \\
\hline KRAS analysis & & CA 19-9 & \\
\hline Mutant & $29(53.7)$ & Median (ng/ml) & 39.5 \\
\hline Wild & $25(46.3)$ & (range) & $(1-15,100)$ \\
\hline \multicolumn{4}{|l|}{$\begin{array}{l}\text { Number of } \\
\text { metastatic site }\end{array}$} \\
\hline 1 & $33(61.1)$ & & \\
\hline$>1$ & 21 (38.9) & & \\
\hline
\end{tabular}

BMI body mass index, CEA carcinoembryonic antigen, CA 19-9 carbohydrate antigen 19-9

clearly established excess body fat as a risk factor for colorectal cancer. Specifically, risk increases with increasing BMI in a sex-(greater risk for men) and site-specific (greater occurrence in the colon versus rectum) manner [21]. A number of plausible biological mechanisms are responsible for the observed associations, including increased insulin resistance, increased availability of insulin-like growth factor (IGF)- 1 (which is mitogenic, proapoptotic, and proangiogenic and increases cell motility), and altered adipokine metabolism. These alterations result in increased leptin, which is mitogenic, anti-apoptotic, and proangiogenic, and decreased adiponectin, which is also anti-angiogenic and anti-inflammatory $[5,22]$.

Obesity is a well-established risk factor for developing CRC [23] and has been associated with increased mortality from colon cancer [24, 25]. Obesity is also associated with a sedentary lifestyle and typical Western diet, which are associated with increased rates of cancer recurrence and death among patients with a history of curative surgical resection for CRC [7, 26, 27]. The patho-physiology is not completely understood but may involve adipose tissue production of adipokines and proangiogenic cytokines, such as VEGF, which may promote cancer growth and dysregulated angiogenesis [13]. All of the studies evaluating the association between body mass and mortality caused by CRC have focused on patients at the time of diagnosis of localised diseases. To our knowledge, only one trial [15] has analysed the influence of obesity on the prognosis of metastatic CRC patients receiving VEGFtargeted therapy.

Obesity is strongly associated with changes in the physiological function of adipose tissue and may cause
Table 2 Treatment details of patients with disease progression

\begin{tabular}{ll}
\hline Characteristic & $n=54(100 \%)$ \\
\hline Primary tumour resection & $45(83.3 \%)$ \\
Yes & $9(16.7 \%)$ \\
No & \\
No. of cycles & 10 \\
Median & $(2-32)$ \\
(range) & \\
Combined drug & $19(35.2 \%)$ \\
Oxaliplatin & $35(64.8 \%)$ \\
Irinotecan & \\
Response to bevacizumab-based therapy & $23(42.6 \%)$ \\
CR + PR & $31(57.4 \%)$ \\
SD + PD &
\end{tabular}

$C R$ complete response, $P R$ partial response, $S D$ stable disease, $P D$ progressive disease

insulin resistance, chronic inflammation, and altered secretion of adipokines. Adipose tissue is now recognised to function as an endocrine and paracrine organ that releases cytokine-like polypeptides responsible for widespread biological effects [28, 29]. In particular, adipocytes produce insulin-like growth factor and multiple angiogenic factors, including VEGF and leptin [30]. Leptin exerts direct angiogenic effects [29, 30], upregulates VEGF mRNA expression [31], and induces VEGF-mediated angiogenesis by colonic epithelial cells [32]. Inflammatory cells infiltrating the adipose tissue and adipose stromal cells also contribute to VEGF production [30,33]. Elevated serum VEGF levels have been reported in overweight and obese patients $[13,14]$.

Visceral fat accumulation has been shown to be associated with colorectal adenoma formation [34], colon carcinogenesis [35], and increased risk for developing colon cancer. The amount of visceral fat independently predicts disease-free survival in patients with resectable CRC [8]. The data strongly suggest that visceral fat may induce the accumulation of protumorigenic factors and be associated with poorer outcomes in patients with CRC.

The role of bevacizumab-based therapy (in combination with conventional chemotherapy) has been established as an option for metastatic CRC. Until now, no predictive biomarker has been available to direct treatment algorithms. Biomarkers are available for other mechanism-based therapies in metastatic CRC, including $\mathrm{K}$-ras status and cetuximab therapy, in which measurement of the predictive biomarker clearly discriminates the subsequent treatment response. While using BMI as a 'lifestyle biomarker' may appear to be unconventional (compared with traditional gene or protein biomarkers), 
Table 3 Logistic regression model of clinical features for predicting time to tumour progression (TTP)
In multivariate analysis, the only independent prognostic factor for TTP was BMI $(p=0.01 ; \mathrm{HR} 4.37 ; 95 \% \mathrm{CI}$ 1.34-14.78) for patients with mCRC treated with combination chemotherapy and bevacizumab

* Mann-Whitney U test

\begin{tabular}{|c|c|c|c|c|c|}
\hline Parameter & $n$ & TTP (months) Median & Min. & Max. & $\begin{array}{l}\text { Univariate analysis* } \\
p \text { value }\end{array}$ \\
\hline BMI, $\mathrm{kg} / \mathrm{m}^{2}$ & & & & & 0.004 \\
\hline$<25$ & 21 & 11.70 & 5.68 & 19.02 & \\
\hline$\geq 25$ & 33 & 6.00 & 1.38 & 17.74 & \\
\hline CEA median $(\mathrm{ng} / \mathrm{ml})$ & & & & & 0.566 \\
\hline$<28$ & 34 & 9.49 & 1.38 & 18.60 & \\
\hline$\geq 28$ & 20 & 7.26 & 3.68 & 19.02 & \\
\hline Age (years) & & & & & 0.264 \\
\hline$<60$ & 27 & 9.49 & 3.25 & 19.02 & \\
\hline$\geq 60$ & 27 & 7.00 & 1.38 & 17.74 & \\
\hline Primary tumour & & & & & 0.618 \\
\hline Intact & 9 & 10.28 & 1.38 & 18.60 & \\
\hline Non-intact & 45 & 7.52 & 2.00 & 19.02 & \\
\hline K-ras mutation status & & & & & 0.683 \\
\hline Wild type & 25 & 9.49 & 1.38 & 17.74 & \\
\hline Mutated & 29 & 7.00 & 3.25 & 19.02 & \\
\hline Chemotherapy & & & & & 0.683 \\
\hline Irinotecan-based & 35 & 8.71 & 3.25 & 19.02 & \\
\hline Oxaliplatin-based & 19 & 7.00 & 1.38 & 18.60 & \\
\hline No. of disease sites & & & & & 0.032 \\
\hline$<2$ & 33 & 10.28 & 2.00 & 19.02 & \\
\hline$\geq 2$ & 21 & 5.72 & 1.38 & 17.74 & \\
\hline
\end{tabular}

lifestyle is used as a predictive factor for treatment response of other cancer types; smoking status is used for directing anti-epidermal growth factor receptor (EGFR) therapy (erlotinib) in non-small cell lung cancer.

The limitations of our study include a relatively small number of patients, limited follow-up duration, singlecentre patient recruitment, and a retrospective design. In addition, the definition of obesity is controversial, and it is unclear whether BMI is the most appropriate measure of obesity. BMI and BSA are crude measures of obesity, and VFA (visceral fat area) or SFA (subcutaneous fat area) may be more appropriate obesity indicators. Computed tomography can be used to accurately assess intra-abdominal fat via measurements of SFA and VFA at the level of the umbilicus [23]. However, in clinical practice, SFA and VFA measurements may be difficult and expensive. BMI is a useful, easy, and non-invasive biomarker. Moreover, there is still a controversion among different centres about dosing of chemotherapy for overweight patients due to the concerns of toxicity [36]. In our study, dosage adjustment of chemotherapeutic agents for patients with BSA over $2 \mathrm{~m}^{2}$ might have confounded the evaluation of treatment efficacy. However, bevacizumab dosing was applied according to the actual patient weight; thus, patients are supposed to receive ideal planned anti-VEGF therapy.

In conclusion, our results provide the evidence that the BMI of patients prior to initiating VEGF-targeted therapy is a simple prognostic factor for patients with metastatic CRC. Further studies may help determine whether the predictive effects of high BMI are related to either a larger distribution volume of VEGF-targeted therapies, the production of high levels of VEGF by visceral fat, or both. Consequently, patients with high BMIs may not benefit from VEGF-targeted therapy or may require higher dosages. If further validation studies with VEGF-targeted therapies corroborate our results, BMI should be incorporated into clinical patient care and stratification schema for future clinical trials. VEGF-targeted therapies should consider physiological parameters related to the patient in addition to pathologic parameters related to the tumour.

Conflict of interest There are no financial disclosures from any authors.

Open Access This article is distributed under the terms of the Creative Commons Attribution License which permits any use, distribution, and reproduction in any medium, provided the original author(s) and the source are credited.

\section{References}

1. Wang Y, Beydoun MA. The obesity epidemic in the United States-gender, age, socioeconomic, racial/ethnic, and geographic characteristics: a systematic review and meta-regression analysis. Epidemiol Rev. 2007;29:6-28. 
2. Pischon T, Lahmann PH, Boeing H, Friedenreich C, Norat T, Tjonneland A, et al. Body size and risk of colon and rectal cancer in the European Prospective Investigation Into Cancer and Nutrition (EPIC). J Natl Cancer Inst. 2006;98(13):920-31.

3. Rapp K, Schroeder J, Klenk J, Stoehr S, Ulmer H, Concin H, et al. Obesity and incidence of cancer: a large cohort study of over 145,000 adults in Austria. Br J Cancer. 2005;93(9):1062-7.

4. Larsson SC, Wolk A. Obesity and colon and rectal cancer risk: a meta-analysis of prospective studies. Am J Clin Nutr. 2007;86(3): 556-65.

5. van Kruijsdijk RC, van der Wall E, Visseren FL. Obesity and cancer: the role of dysfunctional adipose tissue. Cancer Epidemiol Biomarkers Prev. 2009;18(10):2569-78.

6. Meyerhardt JA, Catalano PJ, Haller DG, Mayer RJ, Benson AB 3rd, Macdonald JS, et al. Influence of body mass index on outcomes and treatment-related toxicity in patients with colon carcinoma. Cancer. 2003;98(3):484-95.

7. Dignam JJ, Polite BN, Yothers G, Raich P, Colangelo L, O'Connell MJ, et al. Body mass index and outcomes in patients who receive adjuvant chemotherapy for colon cancer. J Natl Cancer Inst. 2006;98(22):1647-54.

8. Moon HG, Ju YT, Jeong CY, Jung EJ, Lee YJ, Hong SC, et al. Visceral obesity may affect oncologic outcome in patients with colorectal cancer. Ann Surg Oncol. 2008;15(7):1918-22.

9. Trevisan M, Liu J, Muti P, Misciagna G, Menotti A, Fucci F. Markers of insulin resistance and colorectal cancer mortality. Cancer Epidemiol Biomarkers Prev. 2001;10(9):937-41.

10. Reeves GK, Pirie K, Beral V, Green J, Spencer E, Bull D. Cancer incidence and mortality in relation to body mass index in the Million Women Study: cohort study. BMJ. 2007;335(7630):1134.

11. Simkens LHJ, Koopman M, Mol L, Veldhuis GJ, Huinink DTB, Muller EW, Derleyn VA, Teerenstra S, Punt CJA. Influence of body mass index on outcome in advanced colorectal cancer patients receiving chemotherapy with or without targeted therapy. Eur J Cancer. 2011;47:2560-7.

12. Hicklin DJ, Ellis LM. Role of the vascular endothelial growth factor pathway in tumor growth and angiogenesis. J Clin Oncol. 2005;23(5):1011-27.

13. Miyazawa-Hoshimoto S, Takahashi K, Bujo H, Hashimoto N, Saito Y. Elevated serum vascular endothelial growth factor is associated with visceral fat accumulation in human obese subjects. Diabetologia. 2003;46(11):1483-8.

14. Silha JV, Krsek M, Sucharda P, Murphy LJ. Angiogenic factors are elevated in overweight and obese individuals. Int $\mathrm{J}$ Obes (Lond). 2005;29(11):1308-14.

15. Guiu B, Petit JM, Bonnetain F, Ladoire S, Guiu S, Cercueil JP, et al. Visceral fat area is an independent predictive biomarker of outcome after first-line bevacizumab-based treatment in metastatic colorectal cancer. Gut. 2010;59(3):341-7.

16. Kerbel RS. Tumor angiogenesis. N Engl J Med. 2008;358(19): 2039-49.

17. Therasse P, Arbuck SG, Eisenhauer EA, Wanders J, Kaplan RS, Rubinstein L, et al. New guidelines to evaluate the response to treatment in solid tumors. European Organization for Research and Treatment of Cancer, National Cancer Institute of the United States, National Cancer Institute of Canada. J Natl Cancer Inst. 2000;92(3):205-16.

18. Hurwitz H, Fehrenbacher L, Novotny W, Cartwright T, Hainsworth J, Heim W, et al. Bevacizumab plus irinotecan, fluorouracil, and leucovorin for metastatic colorectal cancer. N Engl J Med. 2004;350(23):2335-42.

19. Saltz LB, Clarke S, Diaz-Rubio E, Scheithauer W, Figer A, Wong $\mathrm{R}$, et al. Bevacizumab in combination with oxaliplatin-based chemotherapy as first-line therapy in metastatic colorectal cancer: a randomized phase III study. J Clin Oncol. 2008;26(12):2013-9.
20. Rupnick MA, Panigrahy D, Zhang CY, Dallabrida SM, Lowell $\mathrm{BB}$, Langer $\mathrm{R}$, et al. Adipose tissue mass can be regulated through the vasculature. Proc Natl Acad Sci USA. 2002;99(16): 10730-5.

21. Renehan AG, Tyson M, Egger M, Heller RF, Zwahlen M. Bodymass index and incidence of cancer: a systematic review and meta-analysis of prospective observational studies. Lancet. 2008;371(9612):569-78.

22. Renehan AG, Roberts DL, Dive C. Obesity and cancer: pathophysiological and biological mechanisms. Arch Physiol Biochem. 2008;114(1):71-83.

23. Moghaddam AA, Woodward M, Huxley R. Obesity and risk of colorectal cancer: a meta-analysis of 31 studies with 70,000 events. Cancer Epidemiol Biomarkers Prev. 2007;16(12): 2533-47.

24. Murphy TK, Calle EE, Rodriguez C, Kahn HS, Thun MJ. Body mass index and colon cancer mortality in a large prospective study. Am J Epidemiol. 2000;152(9):847-54.

25. Calle EE, Rodriguez C, Walker-Thurmond K, Thun MJ. Overweight, obesity, and mortality from cancer in a prospectively studied cohort of US adults. N Engl J Med. 2003;348(17): $1625-38$.

26. Meyerhardt JA, Heseltine D, Niedzwiecki D, Hollis D, Saltz LB, Mayer RJ, et al. Impact of physical activity on cancer recurrence and survival in patients with stage III colon cancer: findings from CALGB 89803. J Clin Oncol. 2006;24(22):3535-41.

27. Meyerhardt JA, Niedzwiecki D, Hollis D, Saltz LB, Mayer RJ, Nelson $\mathrm{H}$, et al. Impact of body mass index and weight change after treatment on cancer recurrence and survival in patients with stage III colon cancer: findings from Cancer and Leukemia Group B 89803. J Clin Oncol. 2008;26(25):4109-15.

28. Fox CS, Massaro JM, Hoffmann U, Pou KM, Maurovich-Horvat $\mathrm{P}$, Liu CY, et al. Abdominal visceral and subcutaneous adipose tissue compartments: association with metabolic risk factors in the Framingham heart study. Circulation. 2007;116(1):39-48.

29. Baillargeon J, Rose DP. Obesity, adipokines, and prostate cancer (review). Int J Oncol. 2006;28(3):737-45.

30. Cao Y. Angiogenesis modulates adipogenesis and obesity. J Clin Invest. 2007;117(9):2362-8.

31. Suganami E, Takagi H, Ohashi H, Suzuma K, Suzuma I, Oh H, et al. Leptin stimulates ischemia-induced retinal neovascularization: possible role of vascular endothelial growth factor expressed in retinal endothelial cells. Diabetes. 2004;53(9):2443-8.

32. Birmingham JM, Busik JV, Hansen-Smith FM, Fenton JI. Novel mechanism for obesity-induced colon cancer progression. Carcinogenesis. 2009;30(4):690-7.

33. Rehman J, Traktuev D, Li J, Merfeld-Clauss S, Temm-Grove CJ, Bovenkerk JE, et al. Secretion of angiogenic and antiapoptotic factors by human adipose stromal cells. Circulation. 2004;109(10):1292-8.

34. Otake S, Takeda H, Suzuki Y, Fukui T, Watanabe S, Ishihama K, et al. Association of visceral fat accumulation and plasma adiponectin with colorectal adenoma: evidence for participation of insulin resistance. Clin Cancer Res. 2005;11(10):3642-6.

35. Cowey SL, Quast M, Belalcazar LM, Wei J, Deng X, Given R, et al. Abdominal obesity, insulin resistance, and colon carcinogenesis are increased in mutant mice lacking gastrin gene expression. Cancer. 2005;103(12):2643-53.

36. Griggs JJ, Mangu PB, Anderson H, Balaban EP, Dignam JJ, Hryniuk WM, et al. Appropriate chemotherapy dosing for obese adult patients with cancer: American Society of Clinical Oncology clinical practice guideline. J Clin Oncol. 2012;30(13): 1553-61. 